\title{
Plasma-Enhanced Atomic Layer Deposition of Nanostructured Gold Near Room Temperature
}

\author{
Michiel Van Daele, ${ }^{\dagger} \odot$ Matthew B. E. Griffiths, ${ }^{\dagger}$ Ali Raza, ${ }^{\S, \|}$ Matthias M. Minjauw \\ Ji-Yu Feng, ${ }^{\dagger}$ Ranjith K. Ramachandran, ${ }^{\dagger}{ }^{\oplus}$ Stéphane Clemmen, ${ }^{\S,\|,\|}$ Roel Baets, ${ }^{\S, \|}$ Seán T. Barry, ${ }^{\ddagger}$ \\ Christophe Detavernier, ${ }^{\dagger}{ }^{\dagger}$ and Jolien Dendooven ${ }^{*}{ }^{\dagger} \odot$ \\ ${ }^{\dagger}$ Department of Solid State Sciences, COCOON Group, Ghent University, 9000 Gent, Belgium \\ "Center for Nano- and Biophotonics, Ghent University, 9052 Gent, Belgium \\ ${ }^{\star}$ Department of Chemistry, Carleton University, K1S 5B6 Ottawa, Canada \\ ${ }^{\S}$ Photonics Research Group, INTEC Department, Ghent University-IMEC, 9052 Gent, Belgium \\ ${ }^{\perp}$ ALBA Synchrotron Light Source, NCD-SWEET Beamline, 08290 Cerdanyola del Valles, Spain \\ \#Laboratoire d'Information Quantique, Université Libre de Bruxelles, 1050 Bruxelles, Belgium
} Eduardo Solano, ${ }^{\perp}$

Supporting Information

ABSTRACT: A plasma-enhanced atomic layer deposition (PE-ALD) process to deposit metallic gold is reported, using the previously reported $\mathrm{Me}_{3} \mathrm{Au}\left(\mathrm{PMe}_{3}\right)$ precursor with $\mathrm{H}_{2}$ plasma as the reactant. The process has a deposition window from 50 to $120^{\circ} \mathrm{C}$ with a growth rate of $0.030 \pm 0.002 \mathrm{~nm}$ per cycle on gold seed layers, and it shows saturating behavior for both the precursor and reactant exposure. X-ray photoelectron spectroscopy measurements show that the gold films deposited at $120{ }^{\circ} \mathrm{C}$ are of higher purity than the previously reported ones $(<1$ at. $\%$ carbon and oxygen impurities and $<0.1$ at. $\%$ phosphorous). A low resistivity value was obtained $(5.9 \pm 0.3$

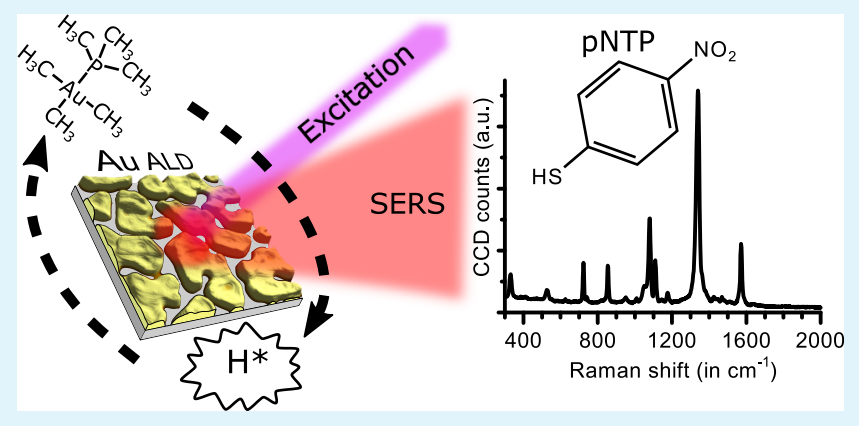
$\mu \Omega \mathrm{cm}$ ), and X-ray diffraction measurements confirm that films deposited at 50 and $120{ }^{\circ} \mathrm{C}$ are polycrystalline. The process forms gold nanoparticles on oxide surfaces, which coalesce into wormlike nanostructures during deposition. Nanostructures grown at $120{ }^{\circ} \mathrm{C}$ are evaluated as substrates for free-space surface-enhanced Raman spectroscopy (SERS) and exhibit an excellent enhancement factor that is without optimization, only one order of magnitude weaker than state-of-the-art gold nanodome substrates. The reported gold PE-ALD process therefore offers a deposition method to create SERS substrates that are template-free and does not require lithography. Using this process, it is possible to deposit nanostructured gold layers at low temperatures on complex three-dimensional (3D) substrates, opening up opportunities for the application of gold ALD in flexible electronics, heterogeneous catalysis, or the preparation of 3D SERS substrates.

KEYWORDS: atomic layer deposition, nanoparticles, plasmonics, SERS, gold metal

\section{INTRODUCTION}

Gold as a bulk material has found a widespread use in jewelry, coinage, and decorative pieces because of its unreactive nature. However, nanoparticulate gold has very interesting and useful catalytic properties and has attracted significant interest for heterogeneous catalysis. ${ }^{1-3}$ The use of nanoparticulate gold for heterogeneous catalysis remains a growing research field.

Suspended gold nanoparticles (or colloidal gold) are often used for their inherent optical properties (e.g., colloidal gold in ruby glass). ${ }^{4}$ The optical properties arise because of the localized surface plasmon resonances (LSPR) that develop at the metal surface. The LSPR can create electromagnetic hotspots between metallic structures, and these hotspots can cause enormous enhancement of a Raman signal. ${ }^{5,6}$ The most used materials for surface-enhanced Raman spectroscopy
(SERS) are silver and gold because of their surface plasmon properties. A drawback of using silver in SERS substrates is that it easily tarnishes while this is not the case for gold. In general, highly ordered nanostructures are required for solidstate SERS substrates. By tuning the properties of the nanostructures on the SERS substrate, it is possible to achieve single molecule detection. A major fallback in present SERS substrates is that fabrication often involves several processing and deposition steps, making the production process expensive, complex, and difficult to implement simply and on a large scale. 
Atomic layer deposition (ALD) offers precise control over the amount of material deposited on a substrate because of the alternating exposure of the substrate to the precursor and reactant gases. These gas phase species undergo self-limiting reactions with the substrate, which allows conformal films to be deposited on planar and complex 3D substrates. This makes ALD an extremely useful deposition method for gold nanoparticles on substrates that are challenging for other deposition methods (e.g., physical vapor deposition or solution-based methods).

Gold metal is extremely challenging to be deposited by ALD: only two gold ALD processes have been reported, although many chemical vapor deposition (CVD) precursors exist to deposit gold. ${ }^{7-11}$ However, finding precursors that are suitable for ALD has proven to be quite difficult because they need to be thermally stable, volatile, have decent surfacelimited reactions, and saturation behavior. ${ }^{12}$ Another aspect is the need for suitable reducing agents for the precursor. The first gold ALD process was reported by Griffiths, Pallister, Mandia, and Barry. ${ }^{13}$ This plasma-enhanced ALD (PE-ALD) process consists of three steps: the surface is first exposed to trimethylphosphinotrimethylgold(III) $\left(\mathrm{Me}_{3} \mathrm{Au}\left(\mathrm{PMe}_{3}\right)\right)$, followed by oxygen plasma exposure, and finally, a water vapor exposure. Deposition of metallic gold was reported at a deposition temperature of $120{ }^{\circ} \mathrm{C}$ with a growth rate of 0.05 $\mathrm{nm}$ per cycle. The deposited films had some impurities, 6.7 at. $\%$ carbon, and 1.8 at. \% oxygen. The second gold ALD process was reported by Mäkelä, Hatanpää, Mizohata, Räisänen, Ritala, and Leskelä. ${ }^{14}$ This process employs $\mathrm{Me}_{2} \mathrm{Au}\left(\mathrm{S}_{2} \mathrm{CNEt}_{2}\right)$ as the gold precursor and ozone as the reactant. Deposition between 120 and $180{ }^{\circ} \mathrm{C}$ was reported, with self-limiting growth at a substrate temperature of $180{ }^{\circ} \mathrm{C}$. A relatively high growth rate of $0.09 \mathrm{~nm}$ per cycle was achieved. These films showed low resistivity $(4.6-16 \mu \Omega \mathrm{cm})$ with some impurities 2.9 at. \% oxygen, 0.9 at. $\%$ hydrogen, 0.2 at. \% carbon, and 0.2 at. \% nitrogen.

In this work, we report a gold PE-ALD process using the existing $\mathrm{Me}_{3} \mathrm{Au}\left(\mathrm{PMe}_{3}\right)$ gold precursor in combination with $\mathrm{H}_{2}$ plasma as the reactant. Compared to the other two reported gold ALD processes, this process showed self-limiting behavior at temperatures as low as $50{ }^{\circ} \mathrm{C}$. This makes it possible to use the reported process in applications that utilize temperaturesensitive substrates, such as flexible electronics. ${ }^{15-17}$ Another advantage over the previously reported gold ALD processes is the use of a reducing coreactant $\left(\mathrm{H}_{2}\right.$ plasma $)$ instead of oxidizing chemistry $\left(\mathrm{O}_{2}\right.$ plasma or $\left.\mathrm{O}_{3}\right)$, hence avoiding the oxidation of the underlying substrate surface. The deposited films have an intrinsic nanoparticle structure, interesting for heterogeneous catalysis and plasmonic applications. It is shown that the films grown at $120{ }^{\circ} \mathrm{C}$ exhibit excellent SERS properties, revealing that the presented PE-ALD process offers a relatively easy route toward large-scale SERS substrates with potential applications in sensing devices. ${ }^{18,19}$

\section{EXPERIMENTAL SECTION}

All ALD processes were carried out in a home-built pump-type ALD reactor with a base pressure of $2 \times 10^{-6} \mathrm{mbar}^{20}$ Computer-controlled pneumatic valves and manually adjustable needle valves were used to control the dose of the precursor vapor and reactant gas. The $\mathrm{Me}_{3} \mathrm{Au}\left(\mathrm{PMe}_{3}\right)$ precursor ( $\geq 95 \%$ purity) was synthesized using the method described in the Supporting Information of the article by Griffiths, Pallister, Mandia, and Barry. ${ }^{13}$ The precursor was kept in a glass container which was heated to $50{ }^{\circ} \mathrm{C}$ during deposition processes, and the delivery line was heated to $55^{\circ} \mathrm{C}$. Argon was used as the carrier gas during all deposition processes. The flow of the carrier gas was adjusted to reach $6 \times 10^{-3} \mathrm{mbar}$ in the chamber when pulsing. The precursor exposure during the ALD processes were carried out by injecting the $\mathrm{Me}_{3} \mathrm{Au}\left(\mathrm{PMe}_{3}\right)$ vapor after closing the gate valve between the turbomolecular pump and the reactor chamber. By varying the injection time, the pressure during the pulse varied between $6 \times 10^{-3}$ and 5 mbar. After injection, the precursor vapor was kept in the ALD chamber for an additional $5 \mathrm{~s}$ before evacuating the chamber. $\mathrm{H}_{2}$ plasma $\left(20 \% \mathrm{H}_{2}\right.$ in argon) was used as the reactant for all deposition processes. Previously, some of the authors reported that using $\mathrm{H}_{2}$ gas or $\mathrm{H}_{2}$ plasma as the reactant in combination with the $\mathrm{Me}_{3} \mathrm{Au}\left(\mathrm{PMe}_{3}\right)$ precursor does not lead to gold deposition. However, they used a low concentration of $\mathrm{H}_{2}$ gas in comparison with the $20 \%$ that was used in this work, possibly explaining this different result. $\mathrm{H}_{2}$ gas was introduced through the plasma column mounted on top of the chamber, and the flow of $\mathrm{H}_{2}$ gas was limited by a needle valve to obtain a chamber pressure of $6 \times 10^{-3} \mathrm{mbar}$ during all deposition processes. A $13.56 \mathrm{MHz}$ radio frequency generator (Advanced Energy, model CESAR 136) and a matching network were used to generate an inductively coupled plasma in the plasma column. For all the experiments, a plasma power of $200 \mathrm{~W}$ was used and the impedance matching parameters were adjusted to minimize the reflected power. $\mathrm{H}_{2}$ plasma exposure of $10 \mathrm{~s}$ was used before each deposition. The used substrates were pieces of p-type silicon (100) with native or thermal silicon oxide or $10 \mathrm{~nm}$ sputtered gold films on p-type silicon (100). The samples were mounted directly on a heated copper block. The temperature of the copper block was adjusted with a proportional-integral-derivative (PID) controller. The chamber walls were heated to $100{ }^{\circ} \mathrm{C}$ for all experiments, except for the experiments to determine the temperature window, for these experiments, the chamber walls were heated to $50{ }^{\circ} \mathrm{C}$. This was necessary to allow the copper block to be heated at temperatures below $80{ }^{\circ} \mathrm{C}$ because it was not possible to use active cooling of the copper block.

Several ex situ measurement techniques were used to determine the physical properties of the deposited Au films. X-ray diffraction (XRD) patterns were acquired to determine the crystallinity of the deposited films. XRD measurements were done on a diffractometer (Bruker D8) equipped with a linear detector (Vantec) and a copper X-ray source ( $\mathrm{Cu} \mathrm{K} \alpha$ radiation). Thickness determination via $\mathrm{X}$-ray reflectivity (XRR) measurements was done on a diffractometer (Bruker D8) equipped with a copper X-ray source $(\mathrm{Cu} \mathrm{K} \alpha$ radiation) and a scintillator point detector. However, because the gold ALD films were generally too rough for accurate thickness determination with XRR, $\mathrm{X}$-ray fluorescence (XRF) measurements were used to determine an equivalent film thickness based on a calibration line of sputtered gold films. The obtained standard deviation of the data points from the obtained calibration line was multiplied by 3 and used as an estimated error for each XRF measurement. The XRF measurements were performed using a Mo X-ray source and an XFlash 5010 silicon drift detectorplaced at an angle of $45^{\circ}$ and $52^{\circ}$ with the sample surface, respectively. An integration time of $200 \mathrm{~s}$ was used to acquire the fluorescence spectra. X-ray photoelectron spectroscopy (XPS) was used to determine the chemical composition and binding energy of the deposited films. The XPS measurements were carried out on a Thermo Scientific Theta Probe XPS instrument. The X-rays were generated using a monochromatic $\mathrm{Al}$ source $(\mathrm{Al} \mathrm{K} \alpha)$. To etch the surface of the deposited films, an $\mathrm{Ar}^{+}$ion gun was used at an acceleration voltage of $3 \mathrm{keV}$ and a current of $2 \mu \mathrm{A}$. An FEI Quanta $200 \mathrm{~F}$ instrument was used to perform scanning electron microscopy (SEM) using secondary electrons and energy-dispersive X-ray spectroscopy (EDX) on the deposited films. Four-point probe measurements were performed to determine the resistivity of the deposited gold films. Atomic force microscopy (AFM) measurements were performed on a Bruker Dimension Edge system to determine the surface roughness of the films. AFM was operated in the tapping mode in air.

To study the morphology of the gold nanostructures, ex situ grazing-incidence small-angle X-ray scattering (GISAXS) measurements were performed at the DUBBLE BM26B beamline of the ESRF 
synchrotron facility. ${ }^{21,22}$ The used energy for the X-ray beam was 12 $\mathrm{keV}$, with an incidence angle of $0.5^{\circ}$. The GISAXS patterns were recorded with a DECTRIS PILATUS3S $1 \mathrm{M}$ detector, which consisted of a pixel array of $1043 \times 981(\mathrm{~V} \times \mathrm{H})$ with a pixel size of $0.172 \times$ $0.172 \mu \mathrm{m}^{2}$, and a sample-detector distance of $4.4 \mathrm{~m}$ was used. The samples were measured in a vacuum chamber that had primary slits and a beamstop inside the chamber to reduce scattering. For each GISAXS scattering pattern, an acquisition time of $60 \mathrm{~s}$ was used. Standard corrections for primary beam intensity fluctuations, solid angle, polarization, and detector efficiency were applied to the collected images. The IsGISAXS software was used to perform the data analysis of the GISAXS scattering patterns; a distorted-wave Born approximation was used and graded interfaces were assumed for the perturbated state caused by the gold particles. A spheroid particle shape was assumed with a Gaussian distribution for the particle size. The particle arrangement on the surface was modeled using a onedimensional (1D) paracrystal model, that is, a $1 \mathrm{D}$ regular lattice with loss of a long-range order. Initial input parameters for the simulation were obtained from the two-dimensional (2D) scattering data, by taking horizontal $\left(q_{y}\right)$ and vertical $\left(q_{z}\right)$ line profiles at the position of the main scattering peak. The maximum in the horizontal line profile gave information about the mean center-to-center particle distance, while information about the particle height was obtained from the minima and maxima observed in the vertical line profile. The input parameters for the simulation were refined until a decent agreement between the experiment and simulation was obtained.

In order to determine the surface enhancement of the deposited gold films, free-space SERS was performed on several samples. A monolayer of 4-nitrothiophenol (pNTP, Sigma) was used as an analyte that selectively binds to the gold surface using a $\mathrm{Au}$-thiol bond. The SERS samples were thoroughly rinsed with acetone, isopropanol, and deionized water and dried using a $\mathrm{N}_{2}$ gun. This was followed by a short $\mathrm{O}_{2}$ plasma exposure, using a PVA-TEPLA GIGAbatch, to remove the remaining contaminants and enhance the binding. The SERS samples were then immersed in $1 \mathrm{mM}$ pNTP solution for $3 \mathrm{~h}$. Finally, the samples were extensively rinsed using ethanol and water to remove unbound pNTP molecules. The number of adsorbed pNTP molecules on the different samples was estimated based on the Au surface area calculations and the reported pNTP density value on $\mathrm{Au}$ (see Supporting Information). Raman measurements were performed using a commercial confocal Raman microscope (WITEC Alpha300R+). A $785 \mathrm{~nm}$ excitation diode laser (Toptica XTRA II) was used as the free-space pump source. The laser was operated at a low pump power of $0.2 \mathrm{~mW}$ to avoid burning or photoreduction of the pNTP molecules. High NA objectives $(100 \times / 0.9$ EC Epiplan Neofluar; $\infty / 0)$ were used to excite the sample and collect the Raman signal. A $100 \mu \mathrm{m}$ multimode fiber was used as a pinhole connected to a spectrometer equipped with a 600 lpmm grating, and a charge-coupled device camera was cooled to -70 ${ }^{\circ} \mathrm{C}$ (Andor iDus 401 BR-DD). All the Raman spectra were acquired after optimizing the $1339 \mathrm{~cm}^{-1}$ peak using an integration time of $1 \mathrm{~s}$.

\section{RESULTS AND DISCUSSION}

3.1. ALD Properties. The reaction of the $\mathrm{Me}_{3} \mathrm{Au}\left(\mathrm{PMe}_{3}\right)$ precursor with $\mathrm{H}_{2}$ plasma was previously reported to not occur. ${ }^{13}$ By using a higher vacuum and higher $\mathrm{H}_{2}$ concentration, this surface reaction was found to proceed in a self-limiting manner. One of the properties of an ALD process is that both reactions show self-limiting behavior. The saturation behavior of $\mathrm{Me}_{3} \mathrm{Au}\left(\mathrm{PMe}_{3}\right)$ and $\mathrm{H}_{2}$ plasma exposure was investigated by determining the equivalent growth per cycle $\left({ }^{\mathrm{eq}} \mathrm{GPC}\right.$, obtained by dividing the equivalent thickness by the number of ALD cycles) on gold seed layers as a function of the respective exposure time (Figure 1). The injection time for the precursor was varied between 1 and $20 \mathrm{~s}$, while the reactant exposure was kept fixed at $20 \mathrm{~s}$. Likewise, the exposure time of the reactant was varied between 1 and $20 \mathrm{~s}$, while the precursor injection time was kept fixed at $20 \mathrm{~s}$. The depositions were

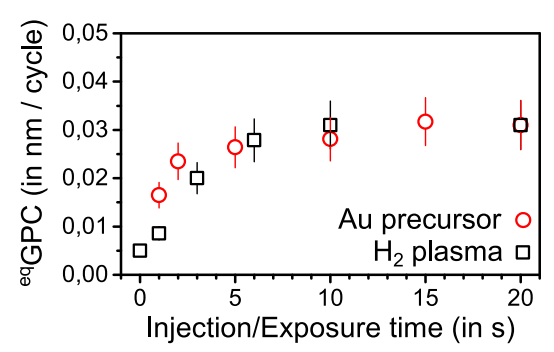

Figure 1. ${ }^{\text {eq }} \mathrm{GPC}$ as a function of the injection time and exposure time for the $\mathrm{Me}_{3} \mathrm{Au}\left(\mathrm{PMe}_{3}\right)$ precursor $(\mathrm{O})$ and $\mathrm{H}_{2}$ plasma $(\square)$, respectively, in the steady growth regime. Depositions were performed on a gold seed layer at a substrate temperature of 100 ${ }^{\circ} \mathrm{C}$. A total of $100 \mathrm{ALD}$ cycles were performed during each deposition to determine the ${ }^{\text {eq }} \mathrm{GPC}$ value. The exposure time of the reactant was kept at $20 \mathrm{~s}$ during the saturation experiments of the precursor. The injection time of the precursor was $20 \mathrm{~s}$ during the saturation experiments of the reactant. The precursor exposure consisted of an injection time that was varied, followed by a fixed dwell time of $5 \mathrm{~s}$.

performed at a substrate temperature of $100{ }^{\circ} \mathrm{C}$ on silicon substrates coated with a thin sputtered gold seed layer (10 $\mathrm{nm}$ ). The gate valve between the reaction chamber and the turbomolecular pump was closed during the precursor exposure. As mentioned in the experimental section, the exposure time consisted of a variable injection time, followed by a fixed dwell time of $5 \mathrm{~s}$. As a result of the varying injection time, the pressure during the precursor exposure varied between $6 \times 10^{-3}$ and $5 \mathrm{mbar}$. As can be seen in Figure 1, saturation was achieved for $\mathrm{Me}_{3} \mathrm{Au}\left(\mathrm{PMe}_{3}\right)$ after an injection time of $10 \mathrm{~s}$ and after an exposure time of $10 \mathrm{~s}$ for $\mathrm{H}_{2}$ plasma, yielding an ${ }^{\mathrm{eq}} \mathrm{GPC}$ of $0.030 \pm 0.002 \mathrm{~nm}$ per cycle in the steady growth regime.

Pulsing the precursor on a gold substrate without any coreactant resulted in an ${ }^{\mathrm{eq}} \mathrm{GPC}$ of $0.005 \mathrm{~nm}$ per cycle, implying a minor CVD component for this ALD process. The monolayer of the adsorbed precursor was most likely not perfectly stable and underwent a very slow decomposition to $\mathrm{Au}(0)$, forming additional adsorption sites for new precursor molecules. Importantly, there was no deposition when exposing a silicon substrate to only the precursor.

Test depositions under thermal conditions were performed using high pressure $\mathrm{H}_{2}$ gas $\left(20 \% \mathrm{H}_{2}\right.$ in argon at $\left.25 \mathrm{mbar}\right)$ instead of $\mathrm{H}_{2}$ plasma as the reactant. An injection time of $15 \mathrm{~s}$ and a dwell time of $5 \mathrm{~s}$ were used for the $\mathrm{Me}_{3} \mathrm{Au}\left(\mathrm{PMe}_{3}\right)$ exposure (i.e., saturating conditions for the PE-ALD process). On silicon substrates, these thermal test depositions did not yield gold deposition in our ALD reactor. However, on gold seed layers some deposition was achieved with an ${ }^{\mathrm{eq}} \mathrm{GPC}$ equal to $0.005 \mathrm{~nm}$ per cycle, likely originating from the abovementioned CVD component rather than a chemical reaction with $\mathrm{H}_{2}$ gas.

The temperature dependence of the ${ }^{\text {eq }} \mathrm{GPC}$ for the PE-ALD process with $\mathrm{H}_{2}$ plasma is shown in Figure 2. The ${ }^{\text {eq }} \mathrm{GPC}$ was determined for two precursor injection times, 10 and $20 \mathrm{~s}$, combined with a $15 \mathrm{~s} \mathrm{H}_{2}$ plasma exposure. Decomposition of the precursor occurred for substrate temperatures above 120 ${ }^{\circ} \mathrm{C}$, as can be concluded from the increase in ${ }^{\text {eq }} \mathrm{GPC}$ at 130 and $140{ }^{\circ} \mathrm{C}$. Although the decomposition remained limited for the lower injection time of $10 \mathrm{~s}$, especially at $130{ }^{\circ} \mathrm{C}$, it was severely increased for the $20 \mathrm{~s}$ injection time. On the other side of the temperature curve, the growth rate remained constant when lowering the substrate temperature. Moreover, over the 
$\mu \mathrm{m}$, a width of $2 \mu \mathrm{m}$, and a center-to-center spacing of $4 \mu \mathrm{m}$, yielding an equivalent aspect ratio (EAR) of 10. The EAR is derived from Monte Carlo simulations and is defined as the aspect ratio of a hypothetical cylindrical hole that would require the same reactant exposure to achieve a conformal coating. ${ }^{24}$ A 15 s exposure time was used for $\mathrm{Me}_{3} \mathrm{Au}\left(\mathrm{PMe}_{3}\right)$ and $10 \mathrm{~s}$ for the $\mathrm{H}_{2}$ plasma exposure during the depositions, performed at a substrate temperature of $120^{\circ} \mathrm{C}$. Using SEM and EDX measurements, the surface morphology and $\mathrm{Au}$ loading were investigated along the length of the pillars (Figure 5). The SEM images (Figure 5a) clearly show that Au was
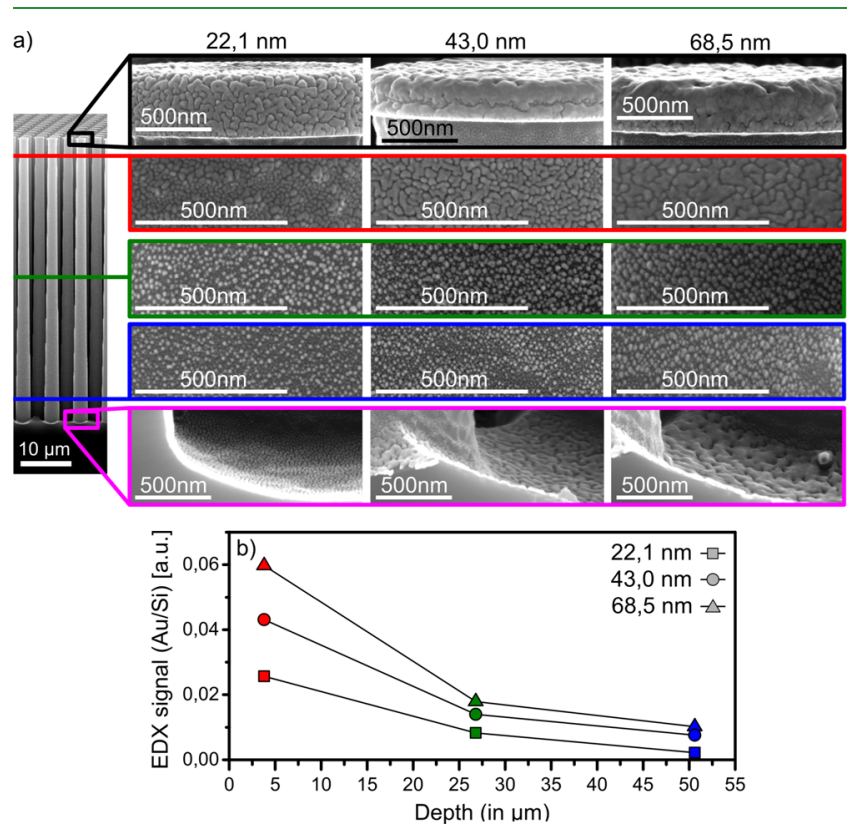

Figure 5. SEM images and EDX signal of gold films, deposited on silicon pillar structures $(\mathrm{EAR}=10)$ at a substrate temperature of 120 ${ }^{\circ} \mathrm{C}$. (a) SEM images for three film thicknesses (as measured on a planar silicon surface): $22.1 \mathrm{~nm}$ ( 800 cycles), $43.0 \mathrm{~nm}$ (1600 cycles), and $68.5 \mathrm{~nm}$ ( 2400 cycles). (b) EDX signal ratio of the Au peak to the $\mathrm{Si}$ peak as a function of distance from the top of the pillar structure.

deposited on the entire substrate and also between the pillars on the bottom surface of the structure. Increasing the thickness of the deposited film resulted in larger particles and more irregular shapes, as expected from the SEM images on planar substrates in Figure $4 \mathrm{~b}, \mathrm{c}$. The morphology of the gold layer changed from being wormlike at the top of the pillar to smaller rounded particles near the bottom, suggesting that the amount of deposited gold on the side walls decreased when going from the top of the pillar to the bottom. To evaluate the Au loading, EDX line scans were taken at the height at which the SEM images were taken, and the ratio of the Au signal to the $\mathrm{Si}$ signal is displayed in Figure $5 b$ as a function of depth in the structure. The data confirms that less gold was present on the side walls deeper in the structure, in agreement with the SEM images. The most likely reason for the nonideal conformality is a too low $\mathrm{H}_{2}$ plasma exposure. Plasma radicals are known to recombine because of surface collisions, thus limiting the conformality, ${ }^{25,26}$ in particular, during metal ALD due to the larger recombination rates on metallic surfaces. ${ }^{27}$ Note that the SEM images visualizing the bottom of the structure and between the pillars revealed wormlike features. This points to a higher Au loading on the area between the pillars than on the bottom region of the pillars' side walls. This can be explained by the fact that the bottom of the structure was in direct line of sight to the plasma, meaning that those surfaces received a larger direct flux of $\mathrm{H}$ radicals than the adjacent walls. Though this "bottom effect" is often predicted by simulation models, ${ }^{24}$ the results presented here provide one of the few experimental examples. Overall, these initial depositions show that it is possible to deposit gold films on 3D structures.

3.2. Physical Properties and Film Composition. XRD measurements were performed on the deposited $\mathrm{Au}$ films to confirm their metallic nature. The obtained XRD patterns for films deposited at 120 and $50{ }^{\circ} \mathrm{C}$ are displayed in Figure $6 \mathrm{a}, \mathrm{b}$.
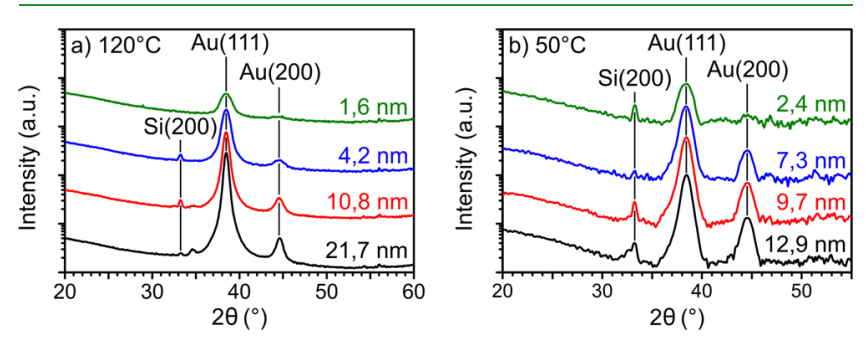

Figure 6. XRD patterns for deposited gold films with an equivalent thickness between 1.6 and $21.7 \mathrm{~nm}$. For all thicknesses, the $\mathrm{Au}(111)$ and $\mathrm{Au}(200)$ peaks are visible at $38.5^{\circ}$ and $44.6^{\circ}$, respectively. The patterns were given an offset for clarity. (a) Films deposited at $120^{\circ} \mathrm{C}$ and (b) films deposited at $50{ }^{\circ} \mathrm{C}$.

The patterns showed that the films were polycrystalline because of the presence of diffraction peaks from the $\mathrm{Au}(111)$ and $\mathrm{Au}(200)$ planes of the cubic gold crystals. These diffraction patterns hint that the as-deposited Au films are polycrystalline for all deposited thicknesses and for the full range of the ALD temperature window.

The composition of the deposited gold films was investigated using XPS measurements. Figure 7 shows the

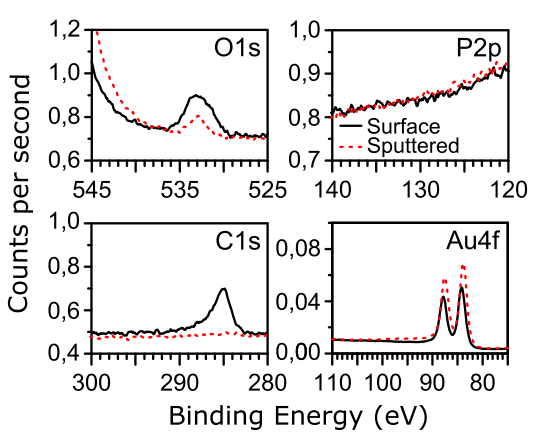

Figure 7. XPS spectra for a PE-ALD-grown Au film deposited at 120 ${ }^{\circ} \mathrm{C}$ with an equivalent thickness of $21.7 \mathrm{~nm}$. The signals are given for the as-deposited film after removing surface contamination by $\mathrm{Ar}$ sputtering. The Si 2 p peak $(99.4 \mathrm{eV})$ is not visible.

Au 4f, C 1s, O 1s, and P 2p spectra. The sample was a silicon substrate on which 800 ALD cycles were performed at $120^{\circ} \mathrm{C}$, yielding an equivalent gold thickness of $21.7 \mathrm{~nm}$. This deposition temperature, at the higher limit of the temperature window, was purposefully selected for comparison with the previously reported gold ALD processes. ${ }^{13,14}$ XPS spectra were measured on the as-deposited film (contaminated by air exposure) and after removing the contaminating top layer by Ar sputtering in the XPS chamber. The surface composition for both cases is given in Table 1 . This shows that the grown films are pure gold films with $<1$ at. \% carbon and oxygen impurities 
Table 1. XPS Concentrations of Au, C, O, and P of a PEALD-Grown Au Film Deposited at $120^{\circ} \mathrm{C}$ with an Equivalent Thickness of $21.7 \mathrm{~nm}^{a}$

$\begin{array}{lcccc} & \mathrm{Au} 4 \mathrm{f}(\text { at. \%) } & \mathrm{C} \text { 1s (at. \%) } & \mathrm{O} \text { 1s (at. \%) } & \mathrm{P} 2 \mathrm{p}(\text { at. \%) } \\ \text { surface } & 95.5 & 3.4 & 1.1 & <0.1 \\ \text { sputtered } & 99.4 & 0.3 & 0.3 & <0.1\end{array}$

${ }^{a}$ The atomic concentration is given for the surface of the (airexposed) as-deposited film on the first row. On the second row the atomic concentration is given after removing the surface contamination from the sample by Ar sputtering in the XPS chamber.

and no phosphorous (below the detection limit, $<0.1$ at. \%) present in the film, values that are clearly lower than those obtained with the previous processes (Griffiths, Pallister, Mandia, and Barry reported 6.7 at. \% carbon and 1.8 at. \% oxygen impurities in their films deposited at $120{ }^{\circ} \mathrm{C}$, and Mäkelä, Hatanpää, Mizohata, Räisänen, Ritala, and Leskelä reported 2.9 at. \% oxygen, 0.9 at. \% hydrogen, 0.2 at. \% carbon, and 0.2 at. \% nitrogen impurities in their films deposited at 180 $\left.{ }^{\circ} \mathrm{C}\right) .{ }^{13,14}$ The lack of phosphorous is a good indication that the $\mathrm{P}\left(\mathrm{CH}_{3}\right)_{3}$ ligands are effectively removed during the ALD surface reactions. After removal of the top layer of the film by argon sputtering, an $\mathrm{O} 1 \mathrm{~s}$ peak remained with a binding energy of $832.8 \mathrm{eV}$, which corresponded to $\mathrm{SiO}_{2}$. Therefore, the likely origin of the $\mathrm{O} 1 \mathrm{~s}$ signal was the $\mathrm{SiO}_{2}$ layer of the substrate. Alternatively, it is possible that minor oxygen contamination in the gold film originated from the glass tube of the plasma column, which may have been slightly etched during the $\mathrm{H}_{2}$ plasma. $^{28}$ The SEM image of the $21.7 \mathrm{~nm}$ thick gold film (Figure 4b) indicates that the film is not a closed layer, and therefore a silicon peak $(99.4 \mathrm{eV})$ was expected, but a clear silicon $2 p$ peak is missing. Because the information depth of XPS is limited to $5-10 \mathrm{~nm}$, the gold film was most likely blocking the silicon substrate from the detector's line of sight. The Au $4 f_{7 / 2}$ peak is located at $84.1 \mathrm{eV}$, close to the expected value of $84.0 \mathrm{eV}$, indicating gold in the metallic state. Furthermore, the Au $4 \mathrm{f}$ region had spin-orbit peaks that were separated in energy by the expected value of $3.7 \mathrm{eV}$.

Although the deposited films had very few impurities and were crystalline, films grown at $120{ }^{\circ} \mathrm{C}$ are not fully closed films at a thickness of $21.7 \mathrm{~nm}$, as indicated by the SEM image in Figure $4 \mathrm{~b}$. The series of SEM images reveals the evolution from isolated circular $\mathrm{Au}$ nanoparticles to larger coalesced wormlike structures. However, the latter does not form a conductive path on the surface. In order to measure the resistivity of the deposited gold films, a thicker film with an equivalent thickness of $65.6 \mathrm{~nm}$ was deposited at $120{ }^{\circ} \mathrm{C}$ on a silicon substrate and four-point probe resistance measurements were performed. For this film, a resistivity value of $5.9 \pm 0.3$ $\mu \Omega \mathrm{cm}$ was obtained. This value is a factor of 2.4 larger than the bulk value of gold $(2.44 \mu \Omega \mathrm{cm})$ and comparable to the resistivity value recently obtained for gold films grown by ALD using the $\mathrm{Me}_{2} \mathrm{Au}\left(\mathrm{S}_{2} \mathrm{CNEt}_{2}\right)$ precursor $(4.6-16 \mu \Omega \mathrm{cm}) .^{14}$ From the top SEM image of the gold film (Figure 8a), it is clear that a percolating film was grown, but there seem to be voids present in the film. Figure $8 \mathrm{~b}$ shows a cross-sectional SEM image on a cleaved edge of the Si substrate, allowing for a visual estimation of the film thickness. The physical thickness varied between 62.0 and $78.1 \mathrm{~nm}$, which is in reasonable agreement with the equivalent thickness of $65.6 \mathrm{~nm}$ (obtained from XRF). Both images show that the deposited films were very rough, with an RMS roughness value of $6.5 \mathrm{~nm}$ obtained

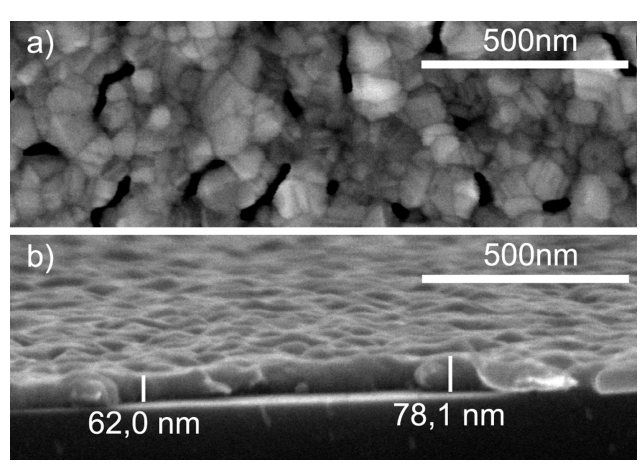

Figure 8. SEM images of a gold film ( 2400 cycles) deposited at 120 ${ }^{\circ} \mathrm{C}$ on a Si substrate with native oxide. The measured equivalent thickness via XRF is $65.6 \mathrm{~nm}$. (a) Top SEM image showing a percolating film with the presence of voids and (b) cross-sectional SEM image on a cleaved edge of the Si substrate.

from AFM measurements (Figure S2). The reason for the higher resistivity, compared to the bulk value of gold, is probably the very rough surface morphology and the presence of holes in the film, lengthening the electrical path.

3.3. Surface Morphology and Raman Spectroscopy. Because of the rough, void-filled nature of the gold films, we speculated that these would be effective SERS substrates. LSPR are needed for a substrate to exhibit SERS properties, and creating very narrow (nanometer-sized) gaps between regular nanostructures made out of $\mathrm{Au}$ or $\mathrm{Ag}$ is a common approach to create LSPR hotspots on a substrate. The enhancement factor (EF) of the SERS signal scales with the inverse of the squared gap-size $\left(d_{\mathrm{g}}\right): \mathrm{EF} \approx 1 / d_{\mathrm{g}}{ }^{2} \cdot{ }^{29}$ The gaps between the PE-ALD deposited gold nanoparticles are of nanometer size, indicating that these can act as LSPR hotspots. To verify this, free-space Raman spectroscopy measurements were performed on a series of four PE-ALD samples deposited at $120{ }^{\circ} \mathrm{C}$, with different gold loadings (Figure $4 \mathrm{~b}$ ). The obtained Raman spectra and the calculated pump to Stokes conversion efficiencies $\left(P_{\mathrm{s}} / P_{\mathrm{p}}\right)$ are shown in Figure 9. The pump to Stokes conversion efficiencies were based on the $1339 \mathrm{~cm}^{-1}$ Raman mode, using the method described by Peyskens, Wuytens, Raza, Van Dorpe, and Baets. ${ }^{30}$ The thinnest sample $(1.6 \mathrm{~nm})$ did not show a decent Raman spectrum of the pNTP molecule while the other samples clearly $\operatorname{did}^{31}$ A stronger Raman signal was observed with increasing equivalent thickness of the PE-ALD gold film. This can also be seen from the trend for the $P_{\mathrm{s}} / P_{\mathrm{p}}$ conversion efficiencies, with the largest increase $(\times 56)$ between the two thinnest samples (1.6 and $4.2 \mathrm{~nm}$ ).

To understand why a stronger Raman signal is measured for the higher gold loadings, it is necessary to determine the surface morphology of the measured samples. Top view SEM images can provide information about the mean particle diameter, gap size, and coverage. SEM images were acquired after the Raman measurement for each sample (Figure 10I) and compared to the SEM images of the as-deposited samples (Figure $4 \mathrm{~b}$ and insets in Figure 10I). This was done to see if the deposited gold nanoparticles remained stable under the processing steps that were needed to bind the pNTP molecules to the surface and the actual Raman measurements. It is easy to see that the two thinnest samples ( $a$ and $b$ ) did not retain their morphology. Instead, the gold nanoparticles agglomerated into irregular clusters, leaving large gaps between the formed clusters. The other two samples seemed to be stable because no agglomerates of particles appeared. The final SEM images 

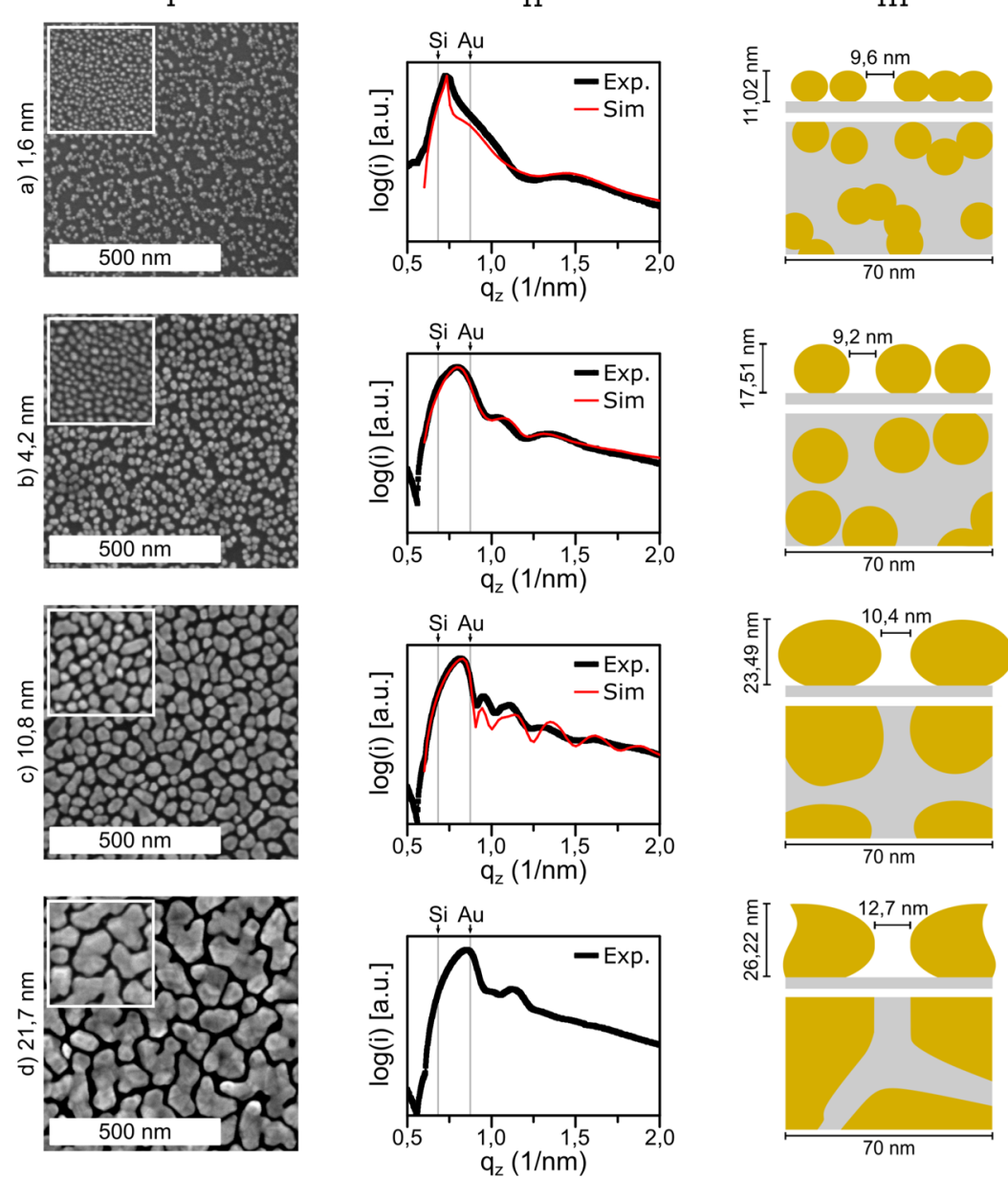

Figure 10. (I) SEM images of the samples after the Raman measurements, the inserts represent the sample before binding pNTP to the Au surface. (II) Vertical cut taken through the scattering maximum of the 2D GISAXS pattern and the simulation result of the cut. (III) Schematic depiction of the side view and the top view of the mean particle gap on each sample.

Table 2. Mean Particle Diameter $\left(d_{\mathrm{p}}\right)$, Gap-Size $\left(d_{\mathrm{g}}\right)$, Particle Height $\left(H_{\mathrm{p}}\right)$, and Pump to Stokes Conversion Efficiency $\left(\boldsymbol{P}_{\mathrm{s}} / \boldsymbol{P}_{\mathrm{p}}\right)$ for Each Sample ${ }^{a}$

$\begin{array}{ccr}\text { sample } & \text { eq. thickness (in } \mathrm{nm}) & d_{\mathrm{g}} \text { (in } \mathrm{nm} \text { ) } \\ \text { (a) } & 1.6 & 9.6 \pm 4.6 \\ \text { (b) } & 4.2 & 9.2 \pm 4.1 \\ \text { (c) } & 10.8 & 10.4 \pm 4.1 \\ \text { (d) } & 21.7 & 12.7 \pm 4.5\end{array}$

$d_{\mathrm{p}}($ in $\mathrm{nm})$
$13.2 \pm 2.9$
$19.9 \pm 4.2$
$36.8 \pm 9.2$
$54.4 \pm 17$

$H_{\mathrm{p}}($ in $\mathrm{nm})$
11.02
17.51
23.49
$(26.22)$

$P_{\mathrm{s}} / P_{\mathrm{p}}$
$9 \times 10^{-12}$
$5 \times 10^{-10}$
$2.5 \times 10^{-9}$
$8.7 \times 10^{-9}$

${ }^{a}$ The standard deviation for the mean particle diameter and gap size are reported next to the tabulated values.

compared to the gap between round particles. This increases the interaction volume where the LSPR hotspots occur, which counteracts the increase in the gap size. The largest gold loading is present on sample (d), and for this sample, the gold nanoparticles have coalesced even more than on sample (c). The resulting nanoparticles form very irregular wormlike shapes. As a result, it is possible for a channel to surround large portions of a particle. This starts to resemble the formation of "racetracks" on the surface, which can exhibit strong SERS enhancements because of their particular morphology. Such types of nanostructures fall in the class of "spoof plasmonics" in which the presence of gaps in a metal can cause LSPR hotspots. ${ }^{37}$ Prokes, Glembocki, Cleveland, Caldwell, Foos, Niinistö, and Ritala demonstrated that this phenomenon can occur in PE-ALD deposited silver thin films because of the formation of "racetrack" structures in the silver thin film. ${ }^{38}$
Here, this particular morphology seems to cause a further increase in the Raman signal, despite the increase of the mean gap size compared to sample (c).

Although the optimal point of the PE-ALD-deposited gold films for SERS enhancement has not been determined, we believe this point must lie somewhere between the surface of sample (d) and a fully closed gold layer. Based on a reported study for sputtered silver films, the best Raman signal is expected at the percolation threshold of the film. ${ }^{39}$ This could also be the case for the PE-ALD-deposited gold films.

To conclude, the strongest Raman signal is obtained for the sample with an equivalent thickness of $21.7 \mathrm{~nm}$ (sample d). Previously, a Stokes to pump conversion efficiency of $6 \times 10^{-8}$ was reported for state-of-the-art gold nanodome substrates. ${ }^{40}$ Correcting for a roughly three times higher accessible gold surface area for the ALD samples (meaning a three times 
higher pNTP concentration), we can conclude that our best sample has a slightly more than one order of magnitude weaker conversion efficiency (factor 21). This is promising, given that there is still room for optimization of the Au ALD films. Although ALD layers have already been used to form protective coatings on SERS substrates and to design the gap of the slot on SiN waveguides for on-chip SERS applications, ${ }^{41,42}$ this work shows that it is possible to create an effective SERS substrate using the reported PE-ALD process, without the need for lithography or a sequence of processing steps.

\section{CONCLUSIONS}

Growth of pure metallic gold films at the lowest reported temperature to date has been demonstrated with a PE-ALD process, using $\mathrm{Me}_{3} \mathrm{Au}\left(\mathrm{PMe}_{3}\right)$ and $\mathrm{H}_{2}$ plasma as the precursor and the reactant, respectively. The process exhibits saturation of the precursor and reactant half cycles on gold seed layers with a steady growth rate of $0.030 \pm 0.002 \mathrm{~nm}$ per cycle. A similar steady growth rate is obtained on bare $\mathrm{SiO}_{2}$ surfaces, after a sufficient number of cycles. Initially, the growth rate is lower because of nucleation, leading to islandlike growth and high film roughness, but percolating films are obtained when the films are sufficiently thick. A resistivity value of $5.9 \pm 0.3$ $\mu \Omega \mathrm{cm}$ is obtained for the thickest films, close to the bulk resistivity value of gold $(2.44 \mu \Omega \mathrm{cm})$. The deposited films are pure gold with $<1$ at. $\%$ carbon and oxygen impurities in the film. The particular nanostructure of as-deposited films offers stable free-space Raman enhancement, slightly more than one order of magnitude lower than that of state-of-the-art solidstate substrates, but with room for further optimization. The SERS-active Au ALD substrates can be fabricated with relative ease without the need for complex-processing or lithography steps. Beyond SERS, nanoparticulate gold has very interesting and useful catalytic properties, ${ }^{1-3}$ making the reported gold nanoparticle ALD process also highly relevant toward heterogeneous catalysis applications.

\section{ASSOCIATED CONTENT}

\section{S Supporting Information}

The Supporting Information is available free of charge on the ACS Publications website at DOI: 10.1021/acsami.9b10848.

Equivalent thicknesses and ${ }^{\mathrm{eq}} \mathrm{GPC}$ for depositions on native and thermal silicon oxide; AFM image of a 65.6 $\mathrm{nm}$ thick Au film on native silicon oxide; 2D GISAXS scattering images of ex situ gold films; cropped SEM image of an ALD Au sample, masks placed over the individual gold particles and border of the individual masks; and estimation procedure for the Au surface area and number of pNTP molecules (PDF)

\section{AUTHOR INFORMATION}

\section{Corresponding Author}

*E-mail: jolien.dendooven@ugent.be.

\section{ORCID}

Michiel Van Daele: 0000-0002-5452-8690

Matthias M. Minjauw: 0000-0003-3620-8949

Eduardo Solano: 0000-0002-2348-2271

Ranjith K. Ramachandran: 0000-0002-9423-219X

Christophe Detavernier: 0000-0001-7653-0858

Jolien Dendooven: 0000-0002-2385-3693
Notes

The authors declare no competing financial interest.

\section{ACKNOWLEDGMENTS}

We are grateful to the ESRF and BM26B staff for smoothly running the synchrotron and beamline facilities. We also thank O. Janssens for performing the SEM measurements. J.D. and R.K.R. thank the FWO-Vlaanderen for a postdoctoral fellowship. The authors thank the FWO for funding. S.T.B. thanks the NSERC for funding (RGPIN-2014-06250).

\section{REFERENCES}

(1) Daniel, M.-C.; Astruc, D. Gold Nanoparticles: Assembly, Supramolecular Chemistry, Quantum-Size-Related Properties, and Applications toward Biology, Catalysis, and Nanotechnology. Chem. Rev. 2004, 104, 293-346.

(2) Hashmi, A. S. K.; Hutchings, G. J. Gold Catalysis. Angew. Chem., Int. Ed. 2006, 45, 7896-7936.

(3) Zhang, Y.; Cui, X.; Shi, F.; Deng, Y. Nano-Gold Catalysis in Fine Chemical Synthesis. Chem. Rev. 2012, 112, 2467-2505.

(4) Freestone, I.; Meeks, N.; Sax, M.; Higgitt, C. The Lycurgus Cup - A Roman Nanotechnology. Gold Bull. 2007, 40, 270-277.

(5) Willets, K. A.; Van Duyne, R. P. Localized Surface Plasmon Resonance Spectroscopy and Sensing. Annu. Rev. Phys. Chem. 2007, $58,267-297$.

(6) Mayer, K. M.; Hafner, J. H. Localized Surface Plasmon Resonance Sensors. Chem. Rev. 2011, 111, 3828-3857.

(7) Hampden-Smith, M. J.; Kodas, T. T. Chemical Vapor Deposition of Metals: Part 1. An Overview of CVD Processes. Chem. Vap. Deposition 1995, 1, 8-23.

(8) Bessonov, A. A.; Morozova, N. B.; Gelfond, N. V.; Semyannikov, P. P.; Trubin, S. V.; Shevtsov, Y. V.; Shubin, Y. V.; Igumenov, I. K. Dimethylgold(III) Carboxylates as new Precursors for Gold CVD. Surf. Coat. Technol. 2007, 201, 9099-9103.

(9) Messelhäuser, J.; Flint, E. B.; Suhr, H. Direct Writing of Gold Lines by Laser-Induced Chemical Vapor Deposition. Appl. Phys. A: Solids Surf. 1992, 55, 196-202.

(10) Baum, T. H.; Jones, C. R. Laser Chemical Vapor Deposition of Gold. Appl. Phys. Lett. 1985, 47, 538-540.

(11) Holl, M. M. B.; Seidler, P. F.; Kowalczyk, S. P.; McFeely, F. R. Surface Reactivity of Alkylgold(I) Complexes: Substrate-Selective Chemical Vapor Deposition of Gold from $\operatorname{RAuP}\left(\mathrm{CH}_{3}\right)_{3} \quad(\mathrm{R}=$ $\mathrm{CH}_{2} \mathrm{CH}_{3}, \mathrm{CH}_{3}$ ) at Remarkably Low Temperatures. Inorg. Chem. 1994, 33, 510-517.

(12) Mäkelä, M.; Hatanpää, T.; Ritala, M.; Leskelä, M.; Mizohata, K.; Meinander, K.; Räisänen, J. Potential Gold(I) Precursors Evaluated for Atomic Layer Deposition. J. Vac. Sci. Technol., A 2017, 35, 01B112.

(13) Griffiths, M. B. E.; Pallister, P. J.; Mandia, D. J.; Barry, S. T. Atomic Layer Deposition of Gold Metal. Chem. Mater. 2016, 28, 4446.

(14) Mäkelä, M.; Hatanpää, T.; Mizohata, K.; Räisänen, J.; Ritala, M.; Leskelä, M. Thermal Atomic Layer Deposition of Continuous and Highly Conducting Gold Thin Films. Chem. Mater. 2017, 29, 61306136.

(15) Gong, S.; Schwalb, W.; Wang, Y.; Chen, Y.; Tang, Y.; Si, J.; Shirinzadeh, B.; Cheng, W. A Wearable and Highly Sensitive Pressure Sensor with Ultrathin Gold Nanowires. Nat. Commun. 2014, 5, 3132.

(16) Fateixa, S.; Pinheiro, P. C.; Nogueira, H. I. S.; Trindade, T. Gold Loaded Textile Fibres as Substrates for SERS Detection. J. Mol. Struct. 2019, 1185, 333-340.

(17) Kim, J.-H.; Twaddle, K. M.; Hu, J.; Byun, H. Sunlight-Induced Synthesis of Various Gold Nanoparticles and Their Heterogeneous Catalytic Properties on a Paper-Based Substrate. ACS Appl. Mater. Interfaces 2014, 6, 11514-11522.

(18) Yu, Y.; Zeng, P.; Yang, C.; Gong, J.; Liang, R.; Ou, Q.; Zhang, S. Gold-Nanorod-Coated Capillaries for the SERS-Based Detection of Thiram. ACS Appl. Nano Mater. 2019, 2, 598-606. 
(19) Kim, H.-M.; Uh, M.; Jeong, D. H.; Lee, H.-Y.; Park, J.-H.; Lee, S.-K. Localized Surface Plasmon Resonance Biosensor using Nanopatterned Gold Particles on the Surface of an Optical Fiber. Sens. Actuators, B 2019, 280, 183-191.

(20) Levrau, E.; Van de Kerckhove, K.; Devloo-Casier, K.; Pulinthanathu Sree, S.; Martens, J. A.; Detavernier, C.; Dendooven, J. In Situ IR Spectroscopic Investigation of Alumina ALD on Porous Silica Films: Thermal versus Plasma-Enhanced ALD. J. Phys. Chem. C 2014, 118, 29854-29859.

(21) Portale, G.; Cavallo, D.; Alfonso, G. C.; Hermida-Merino, D.; van Drongelen, M.; Balzano, L.; Peters, G. W. M.; Goossens, J. G. P.; Bras, W. Polymer Crystallization Studies under Processing-Relevant Conditions at the SAXS/WAXS DUBBLE Beamline at the ESRF. J. Appl. Crystallogr. 2013, 46, 1681-1689.

(22) Dendooven, J.; et al. Independent Tuning of Size and Coverage of Supported Pt Nanoparticles using Atomic Layer Deposition. Nat. Commun. 2017, 8, 1074.

(23) Minjauw, M. M.; Solano, E.; Sree, S. P.; Asapu, R.; Van Daele, M.; Ramachandran, R. K.; Heremans, G.; Verbruggen, S. W.; Lenaerts, S.; Martens, J. A.; Detavernier, C.; Dendooven, J. PlasmaEnhanced Atomic Layer Deposition of Silver Using $\mathrm{Ag}(\mathrm{fod})\left(\mathrm{PEt}_{3}\right)$ and $\mathrm{NH}_{3}$-Plasma. Chem. Mater. 2017, 29, 7114-7121.

(24) Cremers, V.; Puurunen, R. L.; Dendooven, J. Conformality in Atomic Layer Deposition: Current Status Overview of Analysis and Modelling. Appl. Phys. Rev. 2019, 6, 021302.

(25) Dendooven, J.; Deduytsche, D.; Musschoot, J.; Vanmeirhaeghe, R. L.; Detavernier, C. Conformality of $\mathrm{Al}_{2} \mathrm{O}_{3}$ and AlN Deposited by Plasma-Enhanced Atomic Layer Deposition. J. Electrochem. Soc. 2010, 157, G111-G116.

(26) Knoops, H. C. M.; Langereis, E.; van de Sanden, M. C. M.; Kessels, W. M. M. Conformality of Plasma-Assisted ALD: Physical Processes and Modeling. J. Electrochem. Soc. 2010, 157, G241-G249.

(27) Erkens, I. J. M.; Verheijen, M. A.; Knoops, H. C. M.; Keuning, W.; Roozeboom, F.; Kessels, W. M. M. Plasma-Assisted Atomic Layer Deposition of Conformal Pt Films in High Aspect Ratio Trenches. J. Chem. Phys. 2017, 146, 052818.

(28) Chang, R. P. H.; Chang, C. C.; Darack, S. Hydrogen Plasma Etching of Semiconductors and their Oxides. J. Vac. Sci. Technol. 1982, 20, 45-50.

(29) McMahon, J. M.; Li, S.; Ausman, L. K.; Schatz, G. C. Modeling the Effect of Small Gaps in Surface-Enhanced Raman Spectroscopy. J. Phys. Chem. C 2012, 116, 1627-1637.

(30) Peyskens, F.; Wuytens, P.; Raza, A.; Van Dorpe, P.; Baets, R. Waveguide Excitation and Collection of Surface-Enhanced Raman Scattering from a Single Plasmonic Antenna. Nanophotonics 2018, 7, 1299-1306.

(31) Wallace, G. Q.; Tabatabaei, M.; Lagugné-Labarthet, F. Towards Attomolar Detection using a Surface-Enhanced Raman Spectroscopy Platform Fabricated by Nanosphere Lithography. Can. J. Chem. 2014, 92, 1.

(32) Yoneda, Y. Anomalous Surface Reflection of X Rays. Phys. Rev. 1963, 131, 2010-2013.

(33) Holý, V.; Baumbach, T. Nonspecular X-ray Reflection from Rough Multilayers. Phys. Rev. B: Condens. Matter Mater. Phys. 1994, 49, 10668-10676.

(34) Schwartzkopf, M.; et al. From Atoms to Layers: in situ Gold Cluster Growth Kinetics during Sputter Deposition. Nanoscale 2013, 5, 5053-5062.

(35) Bell, S. E. J.; McCourt, M. R. SERS Enhancement by Aggregated Au Colloids: Effect of Particle Size. Phys. Chem. Chem. Phys. 2009, 11, 7455-7462.

(36) Wuytens, P. C.; Skirtach, A. G.; Baets, R. On-Chip SurfaceEnhanced Raman Spectroscopy using Nanosphere-Lithography Patterned Antennas on Silicon Nitride Waveguides. Opt. Express 2017, 25, 12926-12934.

(37) Pendry, J. B.; Martín-Moreno, L.; Garcia-Vidal, F. J. Mimicking Surface Plasmons with Structured Surfaces. Science 2004, 305, 847848.
(38) Prokes, S. M.; Glembocki, O. J.; Cleveland, E.; Caldwell, J. D.; Foos, E.; Niinistö, J.; Ritala, M. Spoof-like Plasmonic Behavior of Plasma Enhanced Atomic Layer Deposition Grown Ag Thin Films. Appl. Phys. Lett. 2012, 100, 053106.

(39) Santoro, G.; Yu, S.; Schwartzkopf, M.; Zhang, P.; Koyiloth Vayalil, S.; Risch, J. F. H.; Rübhausen, M. A.; Hernández, M.; Domingo, C.; Roth, S. V. Silver Substrates for Surface Enhanced Raman Scattering: Correlation between Nanostructure and Raman Scattering Enhancement. Appl. Phys. Lett. 2014, 104, 243107.

(40) Wuytens, P. C.; Subramanian, A. Z.; De Vos, W. H.; Skirtach, A. G.; Baets, R. Gold Nanodome-Patterned Microchips for Intracellular Surface-Enhanced Raman Spectroscopy. Analyst 2015, 140, 8080-8087.

(41) Formo, E. V.; Mahurin, S. M.; Dai, S. Robust SERS Substrates Generated by Coupling a Bottom-Up Approach and Atomic Layer Deposition. ACS Appl. Mater. Interfaces 2010, 2, 1987-1991.

(42) Raza, A.; Clemmen, S.; Wuytens, P.; Muneeb, M.; Van Daele, M.; Dendooven, J.; Detavernier, C.; Skirtach, A.; Baets, R. ALD Assisted Nanoplasmonic Slot Waveguide for On-Chip Enhanced Raman Spectroscopy. APL Photonics 2018, 3, 116105. 